\title{
Automatic Fine Motor Control Behaviours for Autonomous Mobile Agents Operating on Uneven Terrains
}

\author{
Jaime Valls Miro \\ Faculty of Engineering and IT \\ University of Technology Sydney (UTS) \\ Sydney NSW 2007, Australia \\ javalls@eng.uts.edu.au
}

\author{
Gamini Dissanayake \\ Faculty of Engineering and IT \\ University of Technology Sydney (UTS) \\ Sydney NSW 2007, Australia \\ gdissa@eng.uts.edu.au
}

\begin{abstract}
A novel mechanism able to produce increasingly stable paths for mobile robotic agents travelling over uneven terrain is proposed in this paper. In doing so, cognitive agents can focus on higher-level goal planning, with the increased confidence the resulting tasks will be automatically accomplished via safe and reliable paths within the lower-level skills of the platform. The strategy proposes the extension of the Fast Marching level-set method of propagating interfaces in $3 \mathrm{D}$ lattices with a metric to reduce robot body instability. This is particularly relevant for kinematically reconfigurable platforms which significantly modify their mass distribution through posture adaptation, such as humanoids or mobile robots equipped with manipulator arms or varying traction arrangements. Simulation results of an existing reconfigurable mobile rescue robot operating on real scenarios illustrate the validity of the proposed strategy.
\end{abstract}

\section{Categories and Subject Descriptors}

I.2.9 [Artificial Intelligence]: Robotics-autonomous vehicles, kinematics and dynamics

\section{General Terms}

Algorithms, Performance

\section{Keywords}

Path Planning, Autonomous Agents, Cognitive Robots, Uneven Terrain

\section{INTRODUCTION}

For cognitive mobile robots to be safely deployed in the real world they must be able to deal with a large variety of scenarios, predominantly characterised by having to operate in terrains which are far from well-known, flat and homogeneous. The dynamic nature of the environments humans inhabite also mean agent's perceptions need to create a representation of a world around them which is in constant change, with abundant detailed information in some areas, and sparse, uncertain observations in others. These are challenging characteristics on their own which have a strong influence on the robot's ability to perform as planned. In this work, automatic lower-level behaviours are proposed from robot motion first principles to empower intelligent robotic agents with the ability to focus on goal-oriented planning at the higher-level, given the increased certainty the resulting tasks will be executed via safely and reliable paths within the constraints of the platform. This, in effect, follows the abstract model that governs motion in cognitive thinking where fine motor controls are administered spontaneously in nature. For the specific case of reconfigurable robots operating in uneven surfaces, it is most relevant the notion that their kinematic configuration play a crucial factor in the interaction between vehicle and terrain, and having the ability to actively assume safer poses that reduce potential instabilities, such as those leading to vehicle tip-over, is a desirable feature onto which the proposed behaviours is based. Various stability criterions have been proposed in the literature to analyse the qualitative performance of robot stability, mostly with the aim of real-time short-term tip-over monitoring, prediction and prevention, or off-line trajectory optimisation. In this study, a stability measure is also employed to provide a reliable measure for the stability about each tip-over axis of the platform. However, based on this analysis, a novel variational formulation of the classical Fast Marching Method (FMM) is proposed to enhance the safe traversability towards a given goal of the resulting path over irregular terrain. The proposed automatic motion behaviours thus combine the remarkable computational properties of the wavefront propagation proposed by the FMM, with the non-Euclidean metrics derived from the vehicle stability constraints, to extract goal-oriented traversable stable paths for given three-dimensional representations of terrains.

In this work, the proposed planning strategy is illustrated with the quasi-static model of the multi-tracked iRobot PackBot platform, mounted with an arm and a pan and tilt sensor-head unit, as depicted in Figure 1. It is worth noting, however, that the principles are equally applicable to other reconfigurable plaforms with similar kino-dynamic constraints, such as humanoid agents.

The remainder of this paper is organised as follows. Section 2 summarises the mathematical framework of the FMM planner employed in this work. Section 3 reviews the relevant aspects of the proposed stability metric, followed by a description of the robotic platform used in testing in Section 4.Then, the proposed methodology for the solution to planning stable behaviours is presented in Section 5. Experimental results with simulated and real data are provided in Section 6, including a discussion of perceived shortcomings. Finally, Section 7 summarises the contribution of this paper.

\section{LEVEL SET METHODS FOR PATH PLAN- NING}

The Fast Marching Method (FMM) [23] is an efficient numerical method for solving boundary value partial differen- 


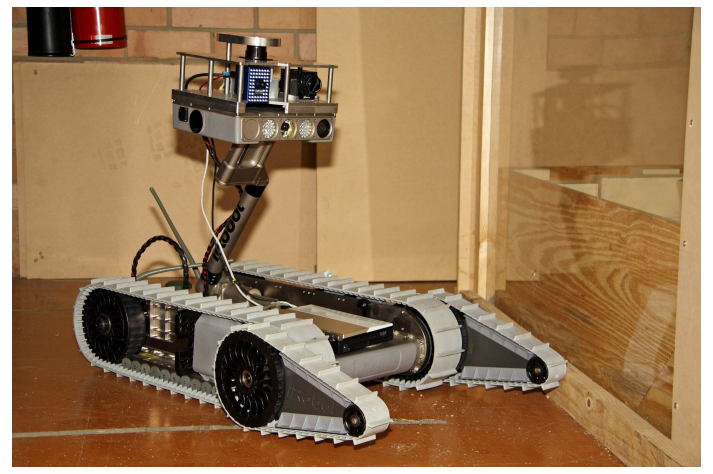

Figure 1: The iRobot PackBot tracked robot fitted with the Explorer arm, and the pan and tilt sensor payload unit.

tial equations in the general context of level set methods for propagating interfaces. The FMM gives the evolution of a continuous front wave in an inhomogeneous medium, whose travel-time is governed by an (approximate) solution to a well-understood non-linear continuous mathematical formula known as the Eikonal equation. The minimal length properties of the resulting geodesic paths have a wide range of applications, including problems in fluid mechanics, combustion, computer animation, image processing, or the structure of snowflakes to name a few.

The extraction of shortest paths has been extensively surveyed in the literature, and it is not the objective of this paper. However, some background is hereby provided, which is by no means comprehensive but for the benefit of contextualising the FMM. Probably the canonical method for computing shortest paths on graphs or discretised settings is Dijkstra's algorithm [4]. To speed up the computations, some heuristics have been proposed that reduced the search space, and the $\mathrm{A}^{*}$ algorithm is extensively used [14], particularly when a path from a given start point to a known goal needs to be calculated in real-time. However, it should be noted that $\mathrm{A}^{*}$ requires different searches for each pair of start and goal points. Other tree-search strategies, such as IDA* [9], have also been proposed. For the case of Euclidean metrics, the exploitation of specific data structures have also given rise to faster algorithms, such as visibility graphs [21]. Despite saving in processing time, in general terms a notable constraint of these discrete-computation methods is the need of "smoothing" operators to produce realistic paths, as the solutions need to follow existing gridding connections. It is clear that the breadth-first nature of the FMM search has an undeniable weakness in the computing overhead when compared with other methods. In contrast, it also exhibits a number of strengths which make it rather attractive to extract geodesics, particularly for three-dimensional data sets : it can be used with non-Euclidean (and continuous) metrics, it can find geodesic paths that follow arbitrary directions, and it is guaranteed to find the optimal paths to each and every one of the units defining the search space, a particularly precious resource in active exploration.

Alternatives to save processing time by heuristically restricting the FMM front propagation between a given pair of points have also been proposed [16].

\subsection{The Fast Marching Method (FMM)}

The following is a brief description of the principal charac- teristics of the FMM. For a detailed description, the reader is referred to [23].

The FMM has been typically applied in problems which deal with evolving fronts, such as seismology. The analogy with seismic theory is rather effective to understand the fundamentals of the FMM [20]. Topographic terrains provide information about elevation of the surface above sea level by contour lines. Each point on a contour line has the same elevation, so a contour line represents an equipotential curve. A set of contour lines tells the trained interpreter the shape of the terrain: hills are represented by concentric loops, stream valleys by vees, steep slopes have closely spaced contour lines, gentle slopes have widely spaced contour lines. The contour interval is the elevation difference between adjacent contour lines. Using an analogy with gravitational fields, the contour lines on a topographic map are lines of constant elevation above sea level and hence of constant gravitational potential energy. If a ball is let free to roll down a mountain, it will follow a path perpendicular to the contour lines, i.e., down the steepest descent or negative gradient. So if contour lines could be measured before releasing the ball, the path the ball would follow down the mountain could be predicted, as the downward path is the curve of steepest slope or negative gradient.

In seismic theory, the travel-time distance surface $T(\mathbf{x})$ is a function of the spatial terrain coordinates that is analogous to the potential, whereas its contour curve represents the propagating wavefronts. The gradient vector is perpendicular to the contour curve, and its magnitude indicates the steepness of the slope. The geodesic curve $\mathbf{r}(t)$ traced out by the seismic energy vector moves in such a manner that its direction at any point coincides with the direction of the gradient at that point. The FMM makes use of the fact that this motion relationship satisfies the non-linear Eikonal equation, which in scalar form is given by:

$$
\|\nabla T(\mathbf{x})\|=s(\mathbf{x})
$$

where the left side implies the wavefront of the potential, and the right side the reciprocal of the seismic velocity, or slowness, a weighting factor dictated by the given terrain.

Hence, given $s(\mathbf{x})$, the weighted geodesic distance between two points $x_{0}, x_{1} \in \mathbb{R}^{d}$ can be defined as

$$
d\left(x_{0}, x_{1}\right)=\min _{r}\left(\int_{0}^{1}\|\mathbf{r}(t)\| s(\mathbf{r}(t)) \mathrm{d} t\right)
$$

where $\mathbf{r}(0)=x_{0}$ and $\mathbf{r}(1)=x_{1}$. It is worth noting that when $s=1$, the integral in (2) corresponds to the length of the curve $\mathbf{r}(t)$ and therefore $d$ is the classical Euclidean distance.

The key feature behind the FMM is a careful selection of the grid points when evaluating the travel time. This order is based on the causality relationship, which states that the arrival time $t$ at any point depends only on the neighbours that have smaller values. During the evolution of the front, each grid point $\mathbf{x}$ is assigned one of the three possible tags:

1. Known: the computed travel time at $\mathbf{x}$ will not be changed later.

2. Narrow-Band (or trial set): the computed travel time at $\mathbf{x}$ may be changed later.

3. Far-Away: the travel time at $\mathbf{x}$ is not yet computed and initially set with infinitely large travel-times. 


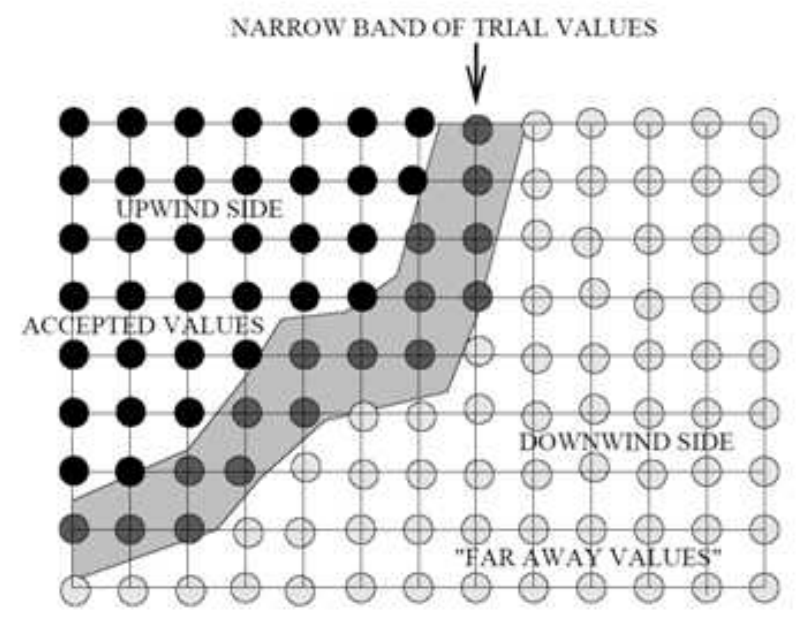

Figure 2: Update of the wavefront propagation time.

as depicted in Figure 2 for a $2 \mathrm{D}$ grid example [5]. The procedure to update $T(\mathbf{x})$ for a given point $\mathbf{x}_{i}$ is based on the upwind first-order approximation to solve (1), a quadratic equation given by:

$$
\sum_{j}=\left(\frac{T\left(\mathbf{x}_{i}\right)-T\left(\mathbf{x}_{j}\right)}{\Delta x_{i j}}\right)^{2}=s^{2}\left(\mathbf{x}_{i}\right)
$$

where $j$ is the (variable) number of neighbouring points and $\Delta x_{i j}$ the grid size in the $i j$ direction (depends on the dimensionality of the problem).

In simple terms, all initial points (one in case of the front emanating from a single point, some other arbitrary shape otherwise) are tagged as Known. Then, their nearest neighbours are tagged as Narrow-Band after computing their arrival time by solving (3) and the fact that a grid's point arrival time gets updated by neighbouring points with smaller travel-times only. This monotonicity property allows for the maintenance of a small Narrow-Band of candidate points around the front representing its outward motion.

As a result of the updating, either a Far-Away point is marked as Narrow-Band, or a Narrow-Band is assigned a new value. When all points have been visited (and unlike other front propagation algorithms each grid point is visited only once [3]), the geodesic curve can then be computed by the back-propagation of the steepest gradient descent. Note that this is a local computation, and only uses the value of $T(\mathbf{x})$ for a small fraction of the visited grid points, all located within the Known set at the end of the front propagation procedure. An example of the evolution of the FMM on a $2 \mathrm{D}$ environment with walls and empty rooms is depicted in Figure 3. It can be observed how the resulting path is not necessarily the safest but the shortest one.

\section{STABILITY METRIC}

There have been a number of propositions to address the issue of stability in mobile robots. Some research has focused on the analysis of the robot's Centre of Gravity (CoG) to find suitable controls to cope with specific scenarios like overcoming obstacles and small ditches [25] or climbing stairs [1].

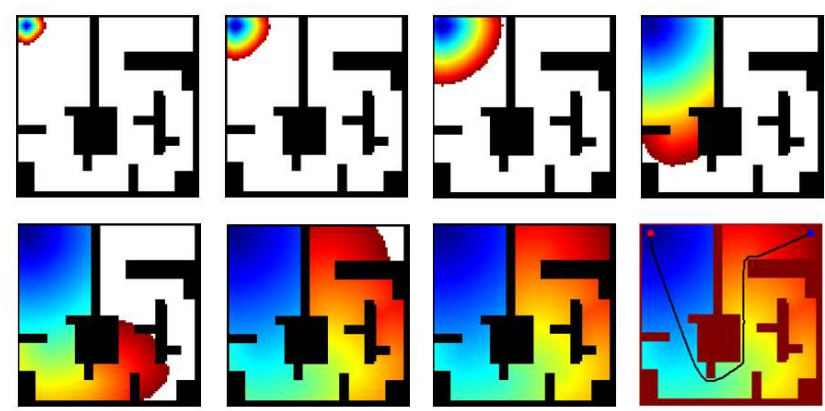

Figure 3: Shortest FMM path on a 2D scenario.

A multi tracked robot on a steep slope was examined in [24] to determine boundaries for the CoG and came up with a strategy to traverse a given slope. A stability margin measure was introduced in [18] to estimate the predicted time until tip-over for large mobile manipulator robots, such as forestry vehicles. They also recommended stabilising steps by using certain actuators. A real-time rollover protection strategy based on whole-body touch sensors that are embedded in the tracks of the robot and an energy stability margin that serves as an indicator for jeopardised robot configurations have also been developed [8]. Stabilising actions to protect the robot from rolling over based upon empirical flipper movement were also proposed.

More general approaches for the stability control of reconfigurable mobile robots which also take into account other constraints such as traction optimisation have been proposed [22], [2]. In both works, the original Force-Angle Stability Margin (FASM) [17] was used. In [22], the performance index considered the stability measure for each potential tip-over axis and the nominal values of the joints. The minimisation of this performance index provided the most favourable configuration of the robot. The combination of the stability measure with an artificial potential field to obtain the demanded actuator values was used in [2]. Both works are however inadequate for certain robot configurations, or in general for robots with low CoG. The stability measure employed considers the angle between the vector through CoG and tip-over axis and the vector of the resulting force through the CoG. This may be sufficient for a robot with relatively high and not significantly changing CoG, but is not representative for the actual stability in many other cases, as will be further discussed in Section 3.1.

Stability Margins have been playing a decisive role in the history of walking robots. The static stability of a walking vehicle were examined for the first time in 1968 [11]. It was claimed that the vehicle was stable if the horizontal projection of the CoG lay within the support polygon that is formed by the contact points between vehicle and ground. The corresponding Static Stability Margin (SSM) was defined as the smallest distance between the projected CoG and the edge of the polygon. The SSM was later adapted to uneven terrain and slightly modified to reduce the complexity of calculation [12]. The main disadvantage of these purely projective-based approaches was the insensitivity to the height of the CoG. The Energy Stability Margin (ESM) [13] solved this problem by determining the potential energy that was needed to tumble the vehicle and represents a reliable static stability margin. This measure 
was normalised [6] to obtain a more general and meaningful measure of stability.

Dynamic effects introduced through accelerations of the whole vehicle or certain components were firstly addressed in [15]. The author presented an extension of the SSM where the CoG was projected onto the support polygon along the vector of the resulting force through the CoG. The resulting forces included dynamic effects, and the system was stable as long as the projection lay within the polygon. Other works referred to this point as the Zero Moment Point [7] where the resulting moment due to reaction forces and moments vanishes. The momentum-based Dynamic Stability Measure [10] was equal to the smallest of all moments about the edges of the support polygon that prevented the vehicle from tipping over, calculated on the basis of reaction forces and moments. The FASM, proposed by Papadopoulos and Rey [19], also covered dynamical changes in the robot configuration and was subject to external forces and moments, but had a more simplistic geometric interpretation and thus could be more easily computed. It constitutes a more suitable stability measure for mobile robots/manipulators and is the metric employed in this work. It was introduced in two different versions, which are briefly reviewed in Section 3.1 to better understand the influence of the CoG's height in the performance of the measure for platforms that can significantly reposition their centre of mass to improve stability in uneven terrains.

\subsection{The Force-Angle Stability Margin (FASM)}

The FASM measure $\beta$ was first proposed in 1996 [17] as

$$
\beta=\min \left(\theta_{i}\left\|\mathbf{f}_{\mathbf{i}}\right\|\right)
$$

where $\mathbf{f}_{\mathbf{i}}$ is the net force (including all static and dynamic forces, as well as moments) contributing to a potential rollover about a particular tip-over axis $\mathbf{a}_{\mathbf{i}}$. The tip-over axes $\mathbf{a}_{\mathbf{i}}$ are given as the lines between $m$ arbitrary supporting points $\mathbf{p}_{\mathbf{i}}, i=\{1, . ., m\}$

$$
\begin{gathered}
\mathbf{a}_{\mathbf{i}}=\mathbf{p}_{\mathbf{i}+\mathbf{1}}-\mathbf{p}_{\mathbf{i}}, i=\{1, . ., m-1\} \\
\mathbf{a}_{\mathbf{m}}=\mathbf{p}_{\mathbf{1}}-\mathbf{p}_{\mathbf{m}}
\end{gathered}
$$

$\theta_{i}$ is the angle between $\mathbf{f}_{\mathbf{i}}$ and the tip-over axis normal through the tip-over axis and the CoG. Figure 4 illustrates these parameters in a two dimensional example, where $\mathbf{a}_{\mathbf{1}}$ and $\mathbf{a}_{\mathbf{3}}$ are perpendicular to the paper representing the tipover axes through $\mathbf{p}_{\mathbf{1}} / \mathbf{p}_{\mathbf{2}}$ and $\mathbf{p}_{\mathbf{3}} / \mathbf{p}_{\mathbf{4}}$ respectively.

The revised version of FASM was published in 2000 [19] and besides $\mathbf{f}_{\mathbf{i}}$ and $\theta_{i}$ also included $\mathbf{d}_{\mathbf{i}}$, the distance between $\mathbf{a}_{\mathbf{i}}$ and $\mathbf{f}_{\mathbf{i}}$ as

$$
\beta=\min \left(\theta_{i}\left\|\mathbf{d}_{\mathbf{i}}\right\|\left\|\mathbf{f}_{\mathbf{i}}\right\|\right)
$$

This enables the metric to become sensitive to varying heights of the CoG. The greater the value of the stability measure $\beta_{i}$, the more stable the vehicle becomes in terms of tipping over about the given axis. Negative values of the measure indicate an occurring tip-over instability.

The tip-over axis normal $\mathbf{l}_{\mathbf{i}}$ that intersects the $\mathrm{CoG}$ is given by

$$
\mathbf{l}_{\mathbf{i}}=\left(I-\hat{\mathbf{a}}_{\mathbf{i}} \hat{\mathbf{a}}_{\mathbf{i}}^{\mathbf{T}}\right)\left(\mathbf{p}_{\mathbf{i}+\mathbf{1}}-\mathbf{p}_{\mathbf{C o G}}\right)
$$

where $\hat{\mathbf{a}}_{\mathbf{i}}$ is the normalised vector of $\mathbf{a}_{\mathbf{i}}, \mathbf{p}_{\mathbf{C o G}}$ is the position of the $\mathrm{CoG}$ and $I$ is the $3 \times 3$ identity matrix.

Given $\mathbf{f}_{\mathbf{r}}$, the net force acting on the CoG which includes gravitational, external and inertial forces, and $\mathbf{n}_{\mathbf{r}}$, the net

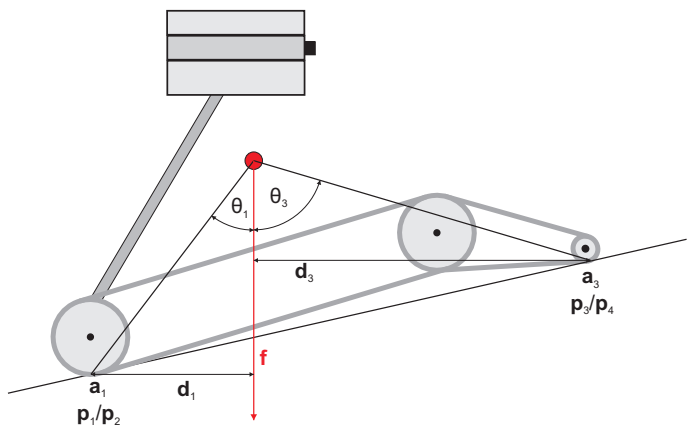

Figure 4: Example FASM in 2D.

moment encompassing all external and inertial moments about the CoG axis, the effective net force $\mathbf{f}_{\mathbf{i}}$ that contributes to a potential tip-over about one specific axis $\mathbf{a}_{\mathbf{i}}$ can be determined by

$$
\mathbf{f}_{\mathbf{i}}=\left(I-\hat{\mathbf{a}}_{\mathbf{i}} \hat{\mathbf{a}}_{\mathbf{i}}^{\mathbf{T}}\right) \mathbf{f}_{\mathbf{r}}+\frac{\hat{\mathbf{l}}_{\mathbf{i}} \times\left(\left(\hat{\mathbf{a}}_{\mathbf{i}} \hat{\mathbf{a}}_{\mathbf{i}}^{\mathbf{T}}\right) \mathbf{n}_{\mathbf{r}}\right)}{\left\|\mathbf{l}_{\mathbf{i}}\right\|}
$$

The first term considers the part of the net force perpendicular to the tip-over axis. The second term considers the moment that participates about the tip-over axis, converted into an equivalent force couple, where one member of the couple passes through the $\mathrm{CoG}$ and thus can be added to the net force, whereas the other member passes through the tip-over axis and hence does not contribute to $\mathbf{f}_{\mathbf{i}}$.

The angle $\theta_{i}$ for each tip-over axis can then be computed by

$$
\theta_{i}=\sigma_{i} \cos ^{-1}\left(\hat{\mathbf{f}}_{\mathbf{i}} \hat{\mathbf{l}}_{\mathbf{i}}\right)
$$

where

$$
\sigma_{i}= \begin{cases}+1 & \left(\hat{\mathbf{f}}_{\mathbf{i}} \times \hat{\mathbf{l}}_{\mathbf{i}}\right) \hat{\mathbf{a}}_{\mathbf{i}}>0 \\ -1 & \text { otherwise }\end{cases}
$$

The revised FASM also requires the shortest distance $\mathbf{d}_{\mathbf{i}}$ between $\mathbf{a}_{\mathbf{i}}$ and $\mathbf{f}_{\mathbf{i}}$, which can be obtained by adding the projection of $\mathbf{l}_{\mathbf{i}}$ on $\mathbf{f}_{\mathbf{i}}$ to negative $\mathbf{l}_{\mathbf{i}}$, i.e.

$$
\mathbf{d}_{\mathbf{i}}=-\mathbf{l}_{\mathbf{i}}+\left(\mathbf{l}_{\mathbf{i}}^{\mathbf{T}} \hat{\mathbf{f}}_{\mathbf{i}}\right) \hat{\mathbf{f}}_{\mathbf{i}}
$$

For more details on these derivations, the reader is referred to $[19]$.

\subsection{Normalisation}

The stability measure for a given configuration is normalised over the most stable robot pose. Normalisation facilitates the general interpretation of the stability measure independently of the vehicle type and permits meaningful comparisons based on the stability measures, for different vehicles or for different terrains, an important factor in this work. The normalised measure $\hat{\beta}_{i}$ is given by:

$$
\hat{\beta}_{i}=\frac{\beta_{i}}{\beta_{\text {norm }}}
$$

where $\beta_{\text {norm }}$ is chosen to be the stability criterion for the vehicle in the horizontal position, with arm and flippers folded for the case of the PackBot robot. 

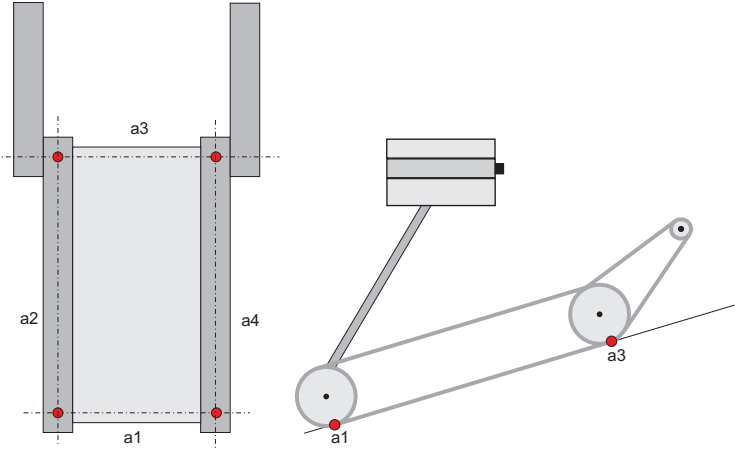

(a) Flippers up contact points.
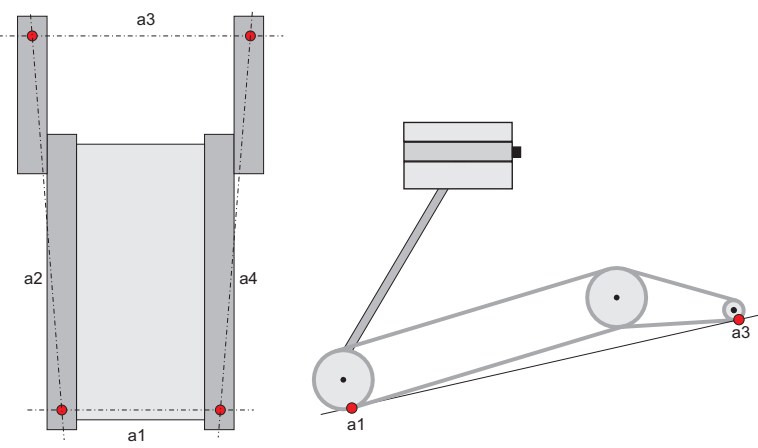

(b) Flippers down contact points.

Figure 5: Surfaces defined by the contact points used to calculate the robot Centre of Gravity.

\section{ROBOT MODEL}

The PackBot robot depicted in Figure 1 was the platform employed to validate the practical aspects of this research. It consists of a skid-steer vehicle base, equipped with two front flippers that enable the robot to traverse obstacles and rough terrain. A manipulator arm attaches to the vehicle base via a 1 Degree of Freedom (DoF) shoulder joint. It carries a 2-DoF pan-and-tilt unit equipped with several cameras and lights. An additional sensor head unit is also mounted on top of the arm head to enhance the Search and Rescue capabilities of the robot in its navigational and victim identification activities. It incorporates a laser scanner, a 3D time-of-flight camera and a thermal camera. The robot is battery powered and features two battery compartments on its left and right hand side. Communication with the operator control unit is wireless.

A modelling of the robot serves as the foundation for the application of the FASM. As expressed in (9), this is captured via the computation of the CoG based on the robot posture and the consideration of the dynamic effects that arise during robot motion. The CoG obtained in the robot frame is given by:

$$
{ }^{R} C o G=\frac{\sum_{i=1}^{n} p_{m_{i}} m_{i}}{m_{\text {tot }}}
$$

where $p_{m, i}$ is the position of the lumped mass $m_{i}$ and $m_{t o t}$ is the total robot mass.

The influence of head panning and tilting on the robot's CoG is very small in comparison to the effects that arise from the position of the arm and flippers, and have therefore been neglected here. Thus, the arm and the flipper poses are the key reconfigurable DoFs considered. While the formulation allows for full dynamic effects to be readily incorporated, as the rover is operated at very low speeds in Search and Rescue operations only results with static forces are presented in this work.

The platform is assumed to make contact with the surface at four equidistant points. They are assumed to be lengthwise symmetrical, depicting two possible convex quadrilateral contact surface as shown in Figure 5. Two contact points are always fixed at the robot base rear sprocket. As flippers operate simultaneously on the PackBot, the other two contacts are chosen based on the flipper pose. When the flippers touch the ground the front contact is at the flipper's front sprockets, defining an isosceles trapezoid as depicted in Figure 5b. When the flippers do not interact with the terrain, the front contact is assumed to be at the robot base front sprockets, thus describing a rectangular area, as shown in Figure 5a. The connecting lines between the ground contacts represent the tip-over axes $a_{i}, i=\{1, . ., 4\}$.

\section{THE STABLE FMM ALGORITHM FOR PLANNING IN 3D LATTICES}

Incorporating the computations for the stability of the robot described by (13) into the FMM path planning framework constitutes the main proposition of this paper. By encouraging the robot to move along paths of higher stability instead of arbitrary metrics, as those illustrated in Figure 6 , the planning stage is shifted towards finding paths that quantitatively aim to guarantee the stability of the robot for a given terrain.

This has been accomplished by merging the stability margins into the FMM potentials through the environmental slowness factor $s(\mathbf{x})$ in (1). The steps are as follows: the set of data points that represent the terrain to be traversed by the robot is first tessellated using Delaunay triangulation (see, for example, Figure 7a). Among other possible triangulation alternatives, Delaunay is preferred for mesh generation as it maximises the minimum triangulation angles. In practice, this means that no data points are contained in the circles circumscribing each triangle. It is assumed in this work that each planar tessellate is sufficiently large to span the robot contact points with the terrain. Having obtained the global virtual slope for each surface in the mesh, the elevation and bank angles of the robot at an arbitrary number of possible orientations within can be calculated for the entire environment, and fed as required to the FASM. Four orientations aligned with the mesh surface have been computed in the results presented here, although finer orientations are equally attainable. An example of the resulting environment potential for the artificial terrain shown in Figure $7 \mathrm{a}$ is depicted in Figure 7c, where colour-coding has been used to visualize the best of the stability margins for the four robot poses considered here, or black if the robot is found to be unstable in the given lattice. Blue equals North, yellow represents South, green is East and red represents West. This terrain representation is then employed by the FMM to propagate the wavefront and find the optimally stable paths. 

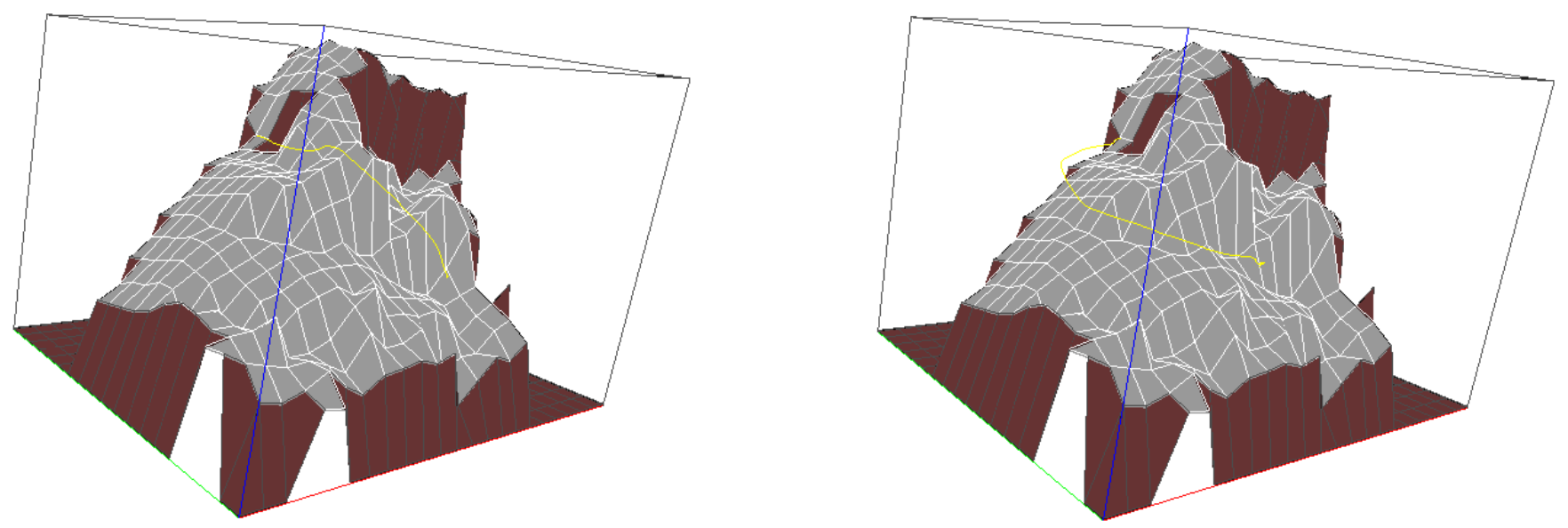

Figure 6: FMM paths (in yellow) on the same 3D real scenario with two potentials: traditional shortest topographical path on the left, and with an arbitrary vertical displacement penalty on the right.

\section{RESULTS AND ANALYSIS}

A couple of examples are provided to show the proposed algorithm in operation. Results depicted in Figure 7 have been artificially created to better illustrate the advantages of the proposed metric. A ramp has been added to a walled environment. It can be seen how the standard wavefront propagation would miss the preferred route, as it is not the shortest path, jeopardising the balance of the platform over the wall. A specific potential for this terrain could also be found that would follow the exit ramp, for instance penalising large discontinuities. However, this is not necessarily a measure applicable to all environments. On the other hand, the proposed methodology is able to exploit robot reconfigurations along the geodesic path in a generalised way to confidently derive stable paths.

The algorithm has also been applied to cloud data obtained from the range camera mounted on the Packbot, deployed in traditional search and rescue test scenarios such as those depicted in Figure 9. Results representative of those obtained are collected in Figure 8, where it can be seen how the proposed planner is able to come up with more stable (if generally longer) paths than those where no stability is accounted for.

The planning mechanism hereby presented constitutes a formalisation of a stability metric to supplement geometric planning. Being intrinsically based on a weighting factor, it is important to raise awareness about the sensitivity of the solution to variations in gridding or the choice of stability scaling, both of which would results in different paths. While the results are encouraging in that suitable stable geodesic paths are computed in a practical manner, this is nevertheless an issue that needs to be further investigated as there is no known generalised solution to overcome this limitation at this stage.

\section{CONCLUSIONS AND FUTURE WORK}

Motion planning behaviours aimed at improving the safe deployment of mobile robotic agents when operating in uneven terrain has been proposed in this work. The ultimate objective is to be able to reliably relinquish higher-level goaloriented cognitive planners of having to account for such low-

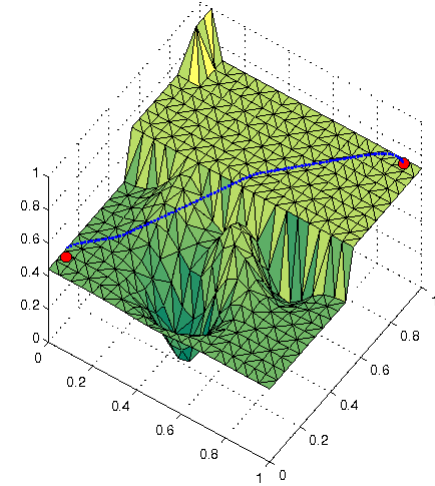

(a) Shortest geodesic.

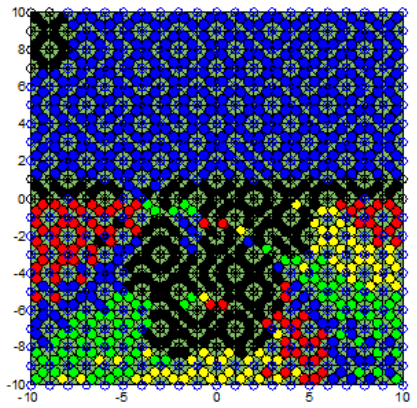

(c) Stability map.

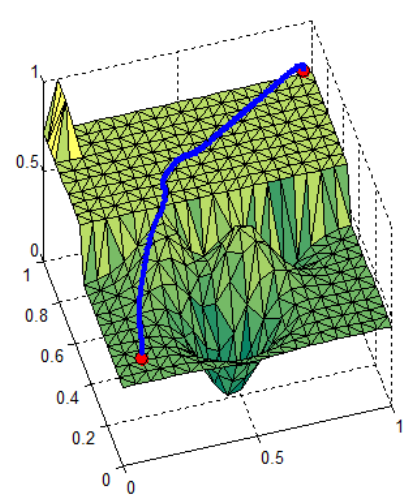

(b) Most stable geodesic 3D.

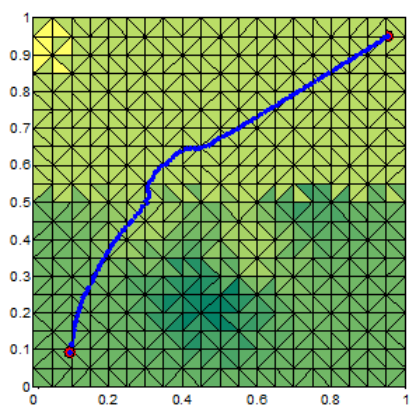
view. (d) Most stable geodesic 2D

Figure 7: Example of stable geodesic paths on a simulated data set. 


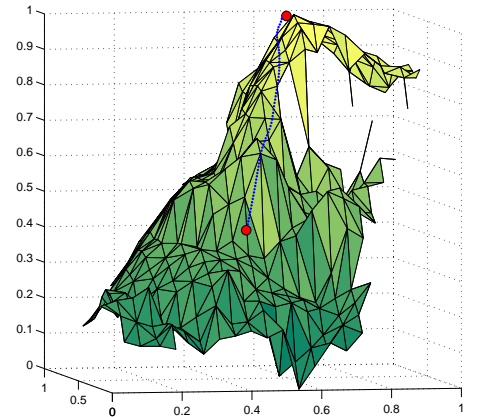

(a) Shortest geodesic.

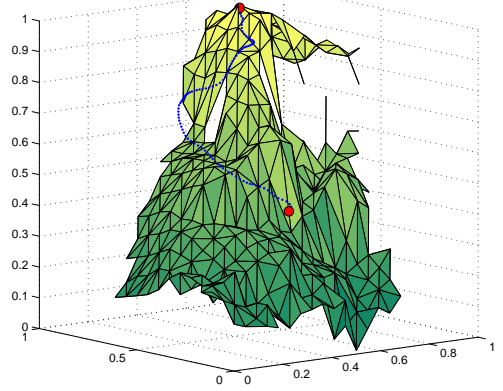

(b) Most stable geodesic.

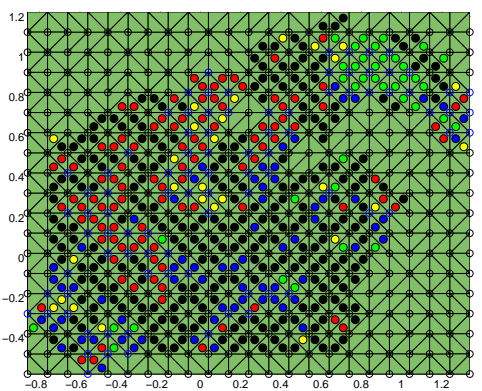

(c) Stability map.

Figure 8: Example of stable geodesic paths on real point cloud.

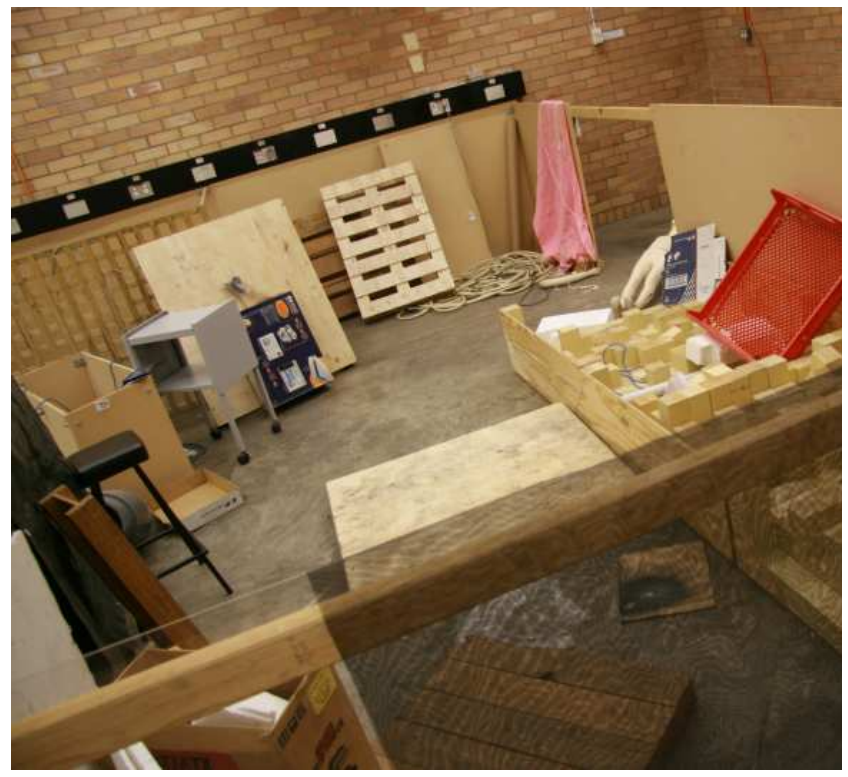

Figure 9: UTS Urban Search and Rescue (USAR) test arena.

level mobility skills in their motion schedules, effectively following the abstract model of human beings where fine motor controls are reactive in nature. The methodology is particular applicable, although not restricted to, reconfigurable platforms that can actively engage in safer poses to reduce potential instabilities, such as those leading to vehicle tipover. This is for instance the case in humanoids, or the large variety of smaller HRI robotic agents endowed with manipulator arms for increased dexterous interaction with environments designed by and for humans. A variational computation based on the Force-Angle Stability Measure (FASM) has been proposed in combination with the Fast Marching level set Method (FMM) to reliably generalise robot instabilities and derive more balanced paths for the robot to follow. Simulations with real and artificial 3D data sets have been provided to demonstrate the performance of the algorithm for a rescue vehicle operating in rough terrain. Future work includes accounting for full dynamics and the addition of other parameters known to jeopardise reliable operations, such as slippage. Integration with a higher-level cognitive planner for a mobile robotic agent fitted with a manipulator arm is also underway within the realm of a typical urban environment where the existence of objects of interest at varying heights, stairs, ramps, curbs, and the like will hopefully demonstrate the effectiveness of the proposed motion planning strategy for enhanced human robot interactions.

\section{ACKNOWLEDGMENTS}

This work is supported by the Australian Research Council $(A R C)$ through its Centre of Excellence programme, and by the New South Wales State Government. The ARC Centre of Excellence for Autonomous Systems $(C A S)$ is a partnership between the University of Technology Sydney, the University of Sydney and the University of New South Wales. The authors would also like to akcnowledge the help of Mr. Christoph Beck and Mr. Gautier Dumonteil in the implementation of this work.

\section{REFERENCES}

[1] P. Ben-Tzvi, S. Ito, and A. A. Goldenberg. Autonomous stair climbing with reconfigurable tracked mobile robot. In ROSE 2007 - IEEE International Workshop on Robotic and Sensors Environments, Ottawa, Canada, 12-13 October 2007, 2007.

[2] G. Besseron, C. Grand, F. B. Amar, and P. Bidaud. Decoupled control of the high mobility robot hylos based on a dynamic stability margin. In IEEE/RSJ International Conference on Intelligent Robots and Systems, Nice, France, September 22-23, 2008.

[3] O. Cuisenaire. Distance Transformations: Fast Algorithms and Applications to Medical Image Processing. PhD thesis, Univ. Catholique de Louvain, Belgium, 1999.

[4] E. W. Dijkstra. A note on two problems in connexion with graphs. Numerische Mathematik, 1:269-271, 1959.

[5] M. Hassouna and A. Farag. Theoretical foundations of tracking monotonically advancing fronts using fast marching level set method. Technical report, Univ. of Louisville, Kentucky, Feb. 2005.

[6] S. Hirose, H. Tsukagoshi, and K. Yoneda. Normalized energy stability margin: generalized stability criterion 
for walking vehicles. Proceedings of the International Conference on Climbing and Walking Robots, Brussels, 1998

[7] Q. Huang and S. Sugano. Manipulator motion planning for stabilizing a mobile-manipulator. International Conference on Intelligent Robots and Systems, 3, 1995.

[8] D. Inoue, K. Ohno, S. Nakamura, and S. Tadokoro. Whole-body touch sensors for tracked mobile robots using force-sensitive chain guides. In Proceedings of the 2008 IEEE International Workshop on Safety, Security and Rescue Robotics, Sendai, Japan, October 2008, 2008.

[9] R. Korf. Depth-first iterative-deepening: An optimal admissible tree search. Artificial Intelligence, 27(1):97-119, 1985.

[10] B.-S. Lin and S.-M. Song. Dynamic modeling, stability and energy efficiency of a quadrupedal walking machine. 1993 IEEE International Conference on Robotics and Automation, May, 1993.

[11] R. McGhee and A. Frank. On the stability properties of quadruped creeping gaits. Mathematical Bioscience 3, 3:331-351, 1968.

[12] R. McGhee and G. Iswandhi. Adaptive locomotion of a multilegged robot over rough terrain. Systems, Man and Cybernetics, 4:176-182, 1979.

[13] D. A. Messuri and C. Klein. Automatic body regulation for maintaining stability of a legged vehicle during rough-terrain locomotion. IEEE Journal of Robotics and Automation, 3:132-141, 1985.

[14] N. Nielsson. Problem-Solving Methods in Artificial Intelligence. McGraw-Hill, 1971.

[15] D. Orin. Interactive control of a six-legged vehicle with optimization of both stability and energy. $P h D$ Thesis, The Ohio State University, 1976.

[16] G. Peyre and L. Cohen. Heuristically driven front propagation for fast geodesic extraction. Copmutational Vision and Biomechanics, 1(1):55-67, 2008.

[17] D. A. Rey and E. G. Papadoupoulos. A new measure of tipover stability margin for mobile manipulators. Proceedings of the IEEE International Conference on Robotics and Automation, Minneapolis, Minnesota, April, 1996.

[18] D. A. Rey and E. G. Papadoupoulos. Online automatic tipover prevention for mobile manipulators. Proceedings of the 1997 IEEE/RSJ International Conference on Intelligent Robots and Systems, 3:1273-1278, 1997.

[19] D. A. Rey and E. G. Papadoupoulos. The force angle measure of tipover stability margin for mobile manipulatiors. Vehicle System Dynamics, 33:29-48, 2000.

[20] E. Robinson and D. Clark. Basic seismology 14 michael faraday and the eikonal equation. The Leading Edge, Society of Exploration Geophysicists, 26(1):24-26, 2007.

[21] H. Rohnert. Shortest path in the plane with convex polygonal obstacles. Information Processing Letters, 23(2):71-76, 1986.

[22] P. Schenker, T. Huntsberger, P. Pirjanian,
S. Dubowsky, K. Iagnemma, and V. Sujan. Rovers for agile, intelligent traverse of challenging terrain. Proceedings of the 7th International Symposium on Artificial Intelligence, Robotics and Automation in Space, i-SAIRAS, Nara, Japan, May, 2003.

[23] J. A. Sethian. Level Set Methods and Fast Marching Methods Evolving Interfaces in Computational Geometry, Fluid Mechanics, Computer Vision, and Materials Science. Cambridge University Press, 1999.

[24] S. Shoval. Stability of a multi tracked robot traveling over steep slopes. Proceedings of the IEEE International Conference on Robotics $\mathcal{B}$ Automation, New Orleans, LA, USA, 2004.

[25] W. Wang, Z. Du, and L. Sun. Dynamic load effect on tracked robot obstacle performance. In Proceedings of International Conference on Mechatronics WA1-B-4, Kumamoto, Japan, 2007. 